\title{
Neue Medien, neue Ökonomie, neue Kultur: Über einige Beiträge zu einer «cultural science» im Zeichen der digitalen Netzwerkkommunikation
}

\author{
Besprochen von VINZENZ HEDIGER
}

1982 schrieb Klaus Kreimeier einen Beitrag für Media, Culture \& Society mit dem Titel Fundamental Reflections on a Materialist Theory of the Mass Media. Darin beklagte er, dass die bourgeoise Kulturtheorie das Werk von cultural mythologists sei und die Produktionsbedingungen von Kultur stets ausblende. "The term «cultural economics»", so Kreimeier in einer erläuternden Klammerbemerkung, «is only to be found in the lexicons of the Socialist countries." Heute, fast dreißig Jahre später, gibt es ein englischsprachiges Journal of Cultural Economics, das von Springer in Wien (ehemals blockfrei) und New York (Welthauptstadt des Kapitalismus) verlegt wird, und an den Wirtschaftsfakultäten rund um die Welt sind immerhin rund 200 Professorinnen und Professoren auf das Studium kultureller und medialer Phänomene unter dem Gesichtspunkt der Frage nach Markt und Preis spezialisiert - nicht viel, wenn man an die in die Zehntausende gehende Gesamtzahl der universitären Ökonomen denkt, aber doch schon deutlich mehr als es an deutschen Universitäten Medienwissenschaftler gibt. Seit kurzem unterhält das Wissenschaftszentrum in Berlin, das Flagschiff der sozialwissenschaftlichen Forschung in der Bundesrepublik, sogar eine eigene Abteilung zur Untersuchung der kulturellen Quellen von Innovation.

Was ist passiert? Haben die Kommunisten eine fünfte Kolonne gebildet und die Wirtschaftswissenschaft unterwandert, mit dem längerfristigen Ziel, von diesem neuen Brückenkopf aus - spät, aber doch noch - die Kulturtheorie zu entbourgeoisieren? Oder haben die «Kulturmythologen», deren Geschäft die Produktion bourgeoiser Kulturtheorie ist, ein Einsehen gehabt und das Feld, das ihnen die sozialistische Gesellschaftstheorie überließ, mit ihrer eigenen Fassung der Analyse der Produktionsmittel besetzt?

Weder noch. Vielmehr war es die Liebe zur Kunst einiger bedeutender Ökonomen, die zum Ferment der neuen Subdisziplin der Kulturökonomik wurde, einer Subdisziplin, die mitunter in eine Neue Medienökonomik übergeht, um den Titel des letzten Buches des Kulturökonomen Michael
Hutter zu zitieren, der die besagte Abteilung am WZB leitet. Der Begriff markiert einen Unterschied zu dem, was in der Publizistik «Medienökonomie» heißt: Das Durchleuchten der Medienindustrien am Leitfaden der Frage, wem welches Medienunternehmen gehört und wer wen kontrolliert. Gemeint ist vielmehr die Untersuchung kultureller und medialer Phänomene hinsichtlich ihrer ökonomischen Aspekte, was auch bedeutet: die Analyse medialer Phänomene unter dem Gesichtspunkt der Frage, inwiefern die klassischen Modelle der Wirtschaftswissenschaften diesen überhaupt noch Rechnung zu tragen vermögen.

Das ist für Medienwissenschaftler deshalb von Interesse, weil die Kultur- und Medienökonomik dort, wo sie in solche Randbereiche vorstößt, interdisziplinär wird und sich unter anderem auf die Medienwissenschaft verwiesen sieht. Seit etwas mehr als zehn Jahren, seit die New Labour-Regierung von Tony Blair TV, Film, Musik, Kunst, Sport und heritage in einem eigenen Ministerialbereich zusammenfasste, laufen die einstigen "Kulturindustrien» und "Bewusstseinsindustrien" unter dem Titel creative industries. Benutzt man, wie mittlerweile in ganz Europa, aber auch in den USA und in Australien üblich, diese kulturpolitische Definition, dann macht die Kultur- und Kreativwirtschaft in westlichen Gesellschaften rund $20 \%$ des gesamten Wirtschaftsaufkommens aus. In Deutschland entspricht dies etwa der Größe der chemischen oder der Automobilindustrie.

Das sind Dimensionen, die an sich schon die Entwicklung eines eigenen Forschungsfeldes rechtfertigen. Es handelt sich aber auch um ein Gegenstandsfeld, das so komplex ist, dass ihm letztlich nur mit einer interdisziplinären Vorgehensweise beizukommen ist. Ein Modell für eine solche Kooperation liefert etwa die Queensland University of Technology in Australien, wo der Medienwissenschaftler John Hartley mit dem Soziologen Stuart Cunningham und dem Ökonomen Jason Potts eine Forschungsgruppe unterhält, die in den letzten Jahren viele der maßgeblichen Beiträge zur wachsenden Literatur über die Kultur- und Kreativwirtschaft geleistet hat. 
Für eine deutschsprachige Leserschaft von Interesse ist dabei nicht zuletzt die Tatsache, dass die beteiligten Forscher ihre Kooperation unter den Titel cultural science stellen, ein Label, das Friedrich Kittler vorübergehend als englische Übersetzung des deutschen Markennamens «Kulturwissenschaft» zu besetzen versuchte.

\section{Der Preis der Kultur}

Die Anfänge der Kultur- und Medienökonomik, die unter den Bedingungen der entfalteten creative industries in eine interdisziplinäre cultural science mündet, liegen in den 196oer Jahren, als sich eine kleine Gruppe von kulturinteressierten Ökonomen für die ökonomischen Aspekte von Kulturpolitik in westlichen Gesellschaften zu interessieren begann. So untersuchte der amerikanische Ökonom William J. Baumol, der in ähnlicher Weise zu den ständigen Anwärtern auf den Wirtschaftsnobelpreis zählt wie Philip Roth zu den Anwärtern auf den Literaturnobelpreis, über einen längeren Zeitraum die Budgets von großen amerikanischen Orchestern. Auf der Grundlage der analysierten Daten diagnostizierte er das, was seither in der Volkswirtschaftslehre "Kostenkrankheit» heißt. ${ }^{1}$ Kulturinstitutionen wie Orchester, so sein Befund, weisen die Eigenheit auf, dass sie bei steigenden Kosten keine Rationalisierungsgewinne erzielen. In herkömmlichen Industrien steigern neue Technologien und Arbeitsformen sukzessive die Produktivität, und auch wenn die Kosten aufgrund von Inflation und anderen Faktoren laufend steigen, entstehen dadurch Einsparungen und größere Gewinnmargen, eben Rationalisierungsgewinne. In Kulturbetrieben hingegen tut sich eine Schere auf. Die Ausbildung von Orchestermusikern dauert Jahre; wer ein Orchester von Rang unterhalten will, muss die talentiertesten Musiker zu einem angemessenen Gehalt einstellen, und wer eine Mahler-Symphonie aufführen will, braucht einhundert oder mehr davon. Nicht zur Verfügung steht die Option, den Musiker durch ein technisches Gerät zu ersetzen und dadurch Einsparungen zu erzielen. Die Kosten steigen, nicht aber die Produktivität.

Die Brisanz von Baumols Befund bestand darin, dass diese Analyse für den Dienstleistungssektor überhaupt gilt. So müssen die Gehälter von Lehrern oder Universitätsprofessoren mit Teuerung und Inflation steigen, ohne dass diese in ihrer Arbeit Produktivitätsgewinne erzielen könnten. Oder zumindest war dies bis vor einigen Jahren noch der Fall. Die neue W-Besoldung an deutschen Universitäten mit ihren tiefen Grundgehältern und fehlenden jährlichen Zulagen ist letztlich nichts anderes als ein groß angelegtes Sozialexperiment: nämlich der Versuch, die Folgen der Kostenkrankheit auf den Verursacher abzuwälzen, und die Zulagen für Drittmitteleinwerbung sind Anreize, in einem Bereich Rationalisierungsgewinne zu erzielen, der solche gar nicht kennt.
Spätestens mit Baumols Theorie der Kostenkrankheit lag auf der Hand, dass der Kultursektor den ernsthaften Ökonomen auch dann interessieren musste, wenn er nicht der Liebe zur klassischen Musik oder anderen Kunstformen huldigte. Die theoretischen Probleme, die Wirtschaftswissenschaftler bekamen, wenn sie sich für Kultur und Medien interessierten, standen damit aber erst am Anfang. So werfen Kulturgüter ein Wertproblem auf. Die klassische politische Ökonomie von Smith bis Marx bemaß den Preis einer Ware nach den Kosten der Rohstoffe und der investierten (bzw. gesellschaftlich notwendigen) Arbeit. Die neoklassische Ökonomietheorie ersetzte diese durch eine relationale Werttheorie, der gemäß der Preis das ist, was in einer Marktsituation für eine bestimmte Ware gezahlt wird - wobei die Kosten der Herstellung nach wie vor ein ausschlaggebendes Kriterium bleiben. Zwischen dem Materialwert eines Van Gogh-Gemäldes und der investierten Arbeitszeit einerseits und dem derzeitigen Handelspreis von 150 Mio. Dollar andererseits allerdings besteht keinerlei substantielle Relation mehr. Was ein Verkaufspreis wie derjenige, den ein Van Gogh-Gemälde derzeit erzielt, aussagt, ist dies: dass es für diese Ware im Grunde keinen Preis gibt. Er drückt das basale Paradox aus, vor das Kulturgüter die Wirtschaftswissenschaft stellt: das Paradox, Kriterien für die Bestimmung des Preises von etwas festlegen zu müssen, dessen Wert sich - ähnlich wie der Wert des Lebens oder der Gesundheit - im Grunde nicht in einem Preis ausdrücken lässt. Nicht von ungefähr heißt ein frühes Buch zur Kulturökonomik von William Grampp aus dem Jahr 1989 Pricing the Priceless.

Mag die Preislosigkeit bei Kulturgütern wie einem Gemälde oder einer Fotografie noch mit der schwierigen Quantifizierbarkeit von Geschmacksurteilen zu tun haben, so zeigt sich bei der medialen Kommunikation, dass das Problem grundlegender Natur ist und von deren Güterstruktur herrührt. So unterscheiden sich Informationsgüter - worunter etwa all das fällt, was in der Medienwissenschaft Programm oder Format genannt wird (ein Film, eine Serie etc.) - von herkömmlichen Gütern in entscheidenden Hinsichten. Informationsgüter kennzeichnen sich dadurch, dass sie stets im Überfluss vorhanden, d. h. nicht knapp sind. Es gibt immer mehr Drehbücher und Romane als publiziert werden (können), und es gibt fast so viele Blogs wie potentielle Leser. Ferner geht es bei Informationsgütern um Zugang und nicht primär um Besitz. Wohl kaufen wir Bücher und Filme, aber wir bezahlen damit nur für die Option, nach unserem Gutdünken Zugang zu dem Text oder dem Werk zu haben. Mit der Einrichtung von Streaming-Plattformen und neuen devices wie dem Kindle, mit dem Druckfaksimiles ganzer Bibliotheken heruntergeladen werden können, erübrigt sich letztlich auch diese Art der Sicherung des Zugriffs auf Informationsgüter. Immerhin brauchen Bücher viel Platz, während der 
Kindle in der Hosentasche Platz hat. Und schließlich zirkulieren Informationsgüter in Netzwerken und communities von Nutzern, anstatt auf herkömmlichen Märkten gehandelt zu werden. ${ }^{2}$

In der Kombination mit den Möglichkeiten, die das Internet eröffnet, führen die Strukturmerkmale von Informationsgütern zu einem grundlegenden Wandel dessen, was wir uns angewöhnt haben, die "Wirtschaft" zu nennen. In seinem Buch The Wealth of Networks (2006), einem der wenigen bislang vorliegenden wissenschaftlichen Werke über die wirtschaftlichen und politischen Aspekte des Internets, die weder in Euphorie noch in frenetischen Dystopismus verfallen und längerfristig Bestand haben werden, zeigt der Jurist und Ökonom Yochai Benkler, dass unter den Bedingungen des Internets vormals marginale Formen der Produktion und der Wertschöpfung in den Mainstream der ökonomischen Aktivität vordringen. Namentlich gilt dies für die Aktivität des Teilens und Mitteilens in sozialen Netwerken. Unter Netzwerkbedingungen stellt sich heraus, dass Produzenten bereit sind, ihre Produkte den Konsumenten ohne formalisiertes Entgelt zur Verfügung zu stellen. Wer bloggt, tut dies in der Hoffnung, Leser zu finden. Wer Videos für YouTube produziert, freut sich über Zuspruch und hofft nicht auf ein Einkommen. Und ganz im Sinne seines eigenen Arguments stellt Benkler sein Buch im Rahmen einer creative commons licence als PDF-Datei zum Herunterladen zur Verfügung, obwohl es in der gedruckten Fassung vom Verleger Yale University Press nach wie vor angeboten wird.

Benkler spricht in diesem Zusammenhang von social production; unter Rückgriff auf Erkenntnisse der Ethnologie wird bisweilen auch der Begriff der "Geschenkökonomie» in Anschlag gebracht. Unter den Bedingungen von Google und YouTube wird aber nicht nur die social production zu einem dominanten Modus der Produktion. Kommunikation selbst wird zu einem Prozess der Wertschöpfung. Das paradoxe Problem, den Preis dessen festzulegen, was keinen Preis hat, stellt sich angesichts der potentiell schrankenlosen Zirkulation von Informationsgütern in digitalen Netzwerken in verschärfter Form. Nicht zuletzt einem Bedürfnis einschlägiger Unternehmen und Investoren entsprechend, hat der ObamaBerater Austan Goolsbee zusammen mit Peter Klenow den Vorschlag gemacht, den Wert von Online-Informationsgütern nach der individuellen Lebenszeit zu bemessen, die in deren Kenntnisnahme investiert wird (Goolsbee/Klenow 2006). Google und YouTube haben dieses Problem indes schon auf einer praktischen Ebene gelöst. Mit ihren Algorithmen generieren die beiden Websites personalisierte Werbung. In den Werbefenstern erscheinen Inserate, von denen man aufgrund von Such- und Sehvorlieben des jeweiligen users annehmen kann, dass sie ihn interessieren (es handelt sich um eine Variation des Programms, das die Kauftipps für return customers bei Amazon generiert). Jeder Klick auf ein Inserat wird den Inserenten verrechnet, und die Einnahmen finanzieren den Betrieb der Portale. Das Prinzip ist immer noch das gleiche wie beim kommerziellen Fernsehen: Fernsehsender sell audiences to advertisers, wie es seit den 1950er Jahren heißt. Google und YouTube schaffen einzig eine ungleich größere Feinverteilung. Für beide Bereiche aber gilt, dass Kommunikation als solche eine Form der Wertschöpfung darstellt: Mit jedem Klick auf ein Inserat schaffe ich Wert, und insofern dieser Wert in die Gewährleistung einer Fortführung der Kommunikation einfließt, könnte man sagen, dass die Netzwerkkommunikation - wie zuvor schon das Fernsehen, nur mit einer größeren Zielgenauigkeit - die Unwahrscheinlichkeit von Kommunikation in den Bereich des ökonomisch Wahrscheinlichen, da Profitablen überführt.

Noch offenkundiger wird der Wertschöpfungscharakter von Kommunikation in der Figur des Prosumers, der längst zu so etwas wie dem romantischen Helden der Kultur der "sozialen Produktion" geworden ist. Sein Loblied singt der Urheberrechtsspezialist Lawrence Lessig in seinem neuen Buch Remix. Making Art and Commerce Thrive in the Hybrid Economy (2008). Das 20. Jahrhundert, so Lessig, war dominiert von "read/only»-Medien wie Radio, Film und Fernsehen. Es war eine Kultur, in der wenige mit vielen kommunizierten und der Zugang zu den Medien der Kommunikation die Überwindung hoher ökonomischer Hürden voraussetzte. Am Beginn des 21. Jahrhunderts hingegen ermöglichte die rasche Verbreitung digitaler Technologien die Entfaltung einer neuen "read/write»-Kultur, in der jeder Empfänger auch Sender und jeder Konsument auch Produzent sein kann: Wer einen Computer mit Internet-Zugang besitzt, kann im Prinzip einen Blog unterhalten oder Videos auf YouTube veröffentlichen. Lessigs Ansatzpunkt ist die Frage nach dem Copyright, und seine Analyse der Remix-Kultur läuft auf ein Plädoyer für eine vollständige Entkriminalisierung von Zweitverwendungen von urheberrechtlich geschütztem Material hinaus. Seine Emphase speist sich aus einem genuin modernen Pathos von Freiheit und Individualität, und in seiner Version erscheint der "prosumer" in der Tat als Widergänger des romantischen Künstlers, der jeden kleinen

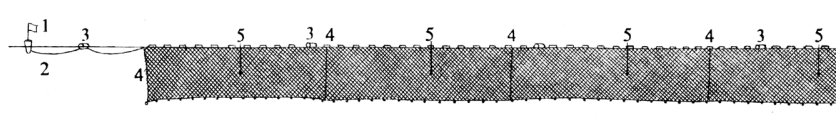


Vorfall zum Anlass künstlerischer Produktivität macht. Mit den kühleren analytischen Arbeiten von Hartley, Potts et al. verbindet Lessig indes sein interdisziplinärer Zugang. Sein medienwissenschaftlicher Hauptzeuge ist Henry Jenkins, einer der Pioniere der Forschung zu Fan-Artefakten, dessen Buch Textual Poachers von 1992 sich mittlerweile liest wie eine Analyse der Remix-Kultur zu Zeiten, als diese ökonomisch gesprochen noch ein unbedeutendes Phänomen war.

Wie sehr das Unterfangen einer kultur- und medienökonomisch orientierten cultural science arbeitsteilig zu bewältigen sein wird, erhellt indes am besten der Titel eines theoretischen Schlüsseltextes, den Hartley, Potts und Cunningham letzten Herbst zusammen mit Paul Ormerod im Journal of Cultural Economics publizierten. Social Network Markets lautet der Hauptitel des Aufsatzes, der sich vornimmt, eine neue Definition der creative industries zu leisten. Nicht mehr das traditionelle Verfahren der Bestimmung von Industrien nach ihrem Input und Output verwenden die Autoren, um die Spezifik der creative industries zu bestimmen. Vielmehr charakterisieren sie die Kreativwirtschaft als Sektor, in dem in kommunikativen Netzwerken Neuheit produziert wird. Michael Hutter spricht in diesem Zusammenhang statt von Input und Output von einer «Neuheitsspirale» (Hutter 2006). Neuheit ist nach einem solchen Ansatz keine Eigenschaft von Produkten oder Dienstleistungen, sondern eine Frage von Zuschreibungen, die in sozialen "Arenen» oder eben Netzwerken stattfinden. Ein Gut zirkuliert, so lange es als neu eingestuft und taxiert wird. Veraltet das Gut, so muss es ersetzt oder aber verbessert werden, um den Kreislauf der Neuheit auf einer weiteren Spirale durchlaufen zu können. Genau das passiert etwa, wenn ein bereits ausgespielter Film als collector's edition auf DVD erscheint und damit eine neue Runde der Zirkulation antreten kann. Die Fokussierung auf die Zuschreibung von Neuheit in Netzwerken als Form der Produktivität ist insofern eine radikale Lösung, als damit der ganze Bereich der Traditionspflege außer Betracht fällt, der gemeinhin zur Kreativwirtschaft gezählt wird, also etwa ausdrücklich auch das Symphonie-Orchester, das doch gerade am Anfang der Entwicklung des Feldes stand. Was an dem Text von Potts et al. innovativ ist, verrät indes schon der Titel Social network markets. Man wird lange suchen müssen, um in einer wirtschaftswissenschaftlichen Fachzeitschrift die Begriffe "social» und "network» in der Kombination mit "markets» in einem Aufsatztitel zu finden. Wirtschaftswissenschaftliche Marktmodelle basieren normalerweise auf der Annahme, dass Markttransaktionen von rational handelnden Individuen vollzogen werden, die auf die Optimierung ihres Eigennutzens bedacht sind. «Soziale Netzwerke» kommen in diesen Modellen nicht vor. Wenn Ökonomen wie Potts und Hutter davon sprechen, dass es Märkte gibt, die als soziale Netzwerke strukturiert sind, und wenn ein Ökonom wie Potts, um diese Märkte mo- dellieren zu können, Soziologen und Medienwissenschaftler zu Rate ziehen muss, dann ist in der Tat der Punkt erreicht, an dem die Wirtschaftswissenschaft zugeben muss, dass sie es mit Gegenständen zu tun hat, bei denen ihre traditionellen theoretischen Modelle an die Grenze ihrer Erklärungskraft stoßen. Es wird auch und gerade für Medienwissenschaftler in den nächsten Jahren von größtem Interesse sein, was in diesem Grenzbereich einer sich abzeichnenden neuen cultural science weiter passiert - und was sie zur Entwicklung dieses Feldes beitragen können.

$1 \mathrm{Vgl}$. William J. Baumol, Macroeconomics of Unbalanced Growth: The Anatomy of Urban Crisis, in: American Economic Review 57, 1967, 416-426. 2 Vgl. hierzu Michael Hutter, Information Goods, in: Ruth Towse (Hg.), A Handbook of Cultural Economics. Cheltenham (Edward Elgar) 2003, $263-268$.

\section{Literatur:}

John Banks, Jason Potts, A Cultural Science of Social Network Markets: Agent-Based Computational Modeling of Online Co-Creative Networks, in: Proceedings of the WebSci'og: Society On-Line, 18.-20.3.2009, Athen, (im Druck).

Yochai Benkler, The Wealth of Networks. How Social Production Transforms Markets and Freedom. New Haven (Yale University Press) 2006 (download mit einer Creative Commons Licence unter http://www.benkler.org/Benkler_Wealth_Of_Networks.pdf).

Austan Goolsbee, Peter Klenow, Valuing Consumer Goods by the Time Spent Using Them. An Application to the Internet, in: American Economic Review (Papers and Proceedings), 96, 2, 2006, 108-113 (http://faculty.chicagobooth.edu/austan. goolsbee/research/time use.pdf).

William J. Grampp, Pricing the Priceless. Art, Artists, and Economics, New York (Basic Books) 1989.

Michael Hutter, Neue Medienökonomik, München (Fink) 2006.

Lawrence Lessig, Remix. Making Art and Commerce Drive in the Hybrid Economy, New York (Penguin) 2008.

Jason Potts, Stuart Cunningham, John Hartley und Paul Ormerod, Social Network Markets: A New Definition of the Creative Industries, in: Fournal of Cultural Economis, 32, 3, 2008, $167-185$. 